\title{
INFLUENCE DE LA SURDITÉ SUR LE DÉVELOPPEMENT CORPOREL ET SEXUEL DE GALLUS DOMESTICUS
}

\author{
J. M. FAURE \\ Station Expérimentale d'Aviculture du Magneraud, I. N.R. A. \\ 17700 Surgères
}

\section{RÉSUMÉ}

L'influence de la surdité sur différentes caractéristiques zootechniques a été étudiée dans une souche de pondeuses de format moyen.

La surdité agit sur la croissance du coq et de la poule en augmentant le poids adulte sans que cette différence puisse ètre attribuée uniquement à une accumulation de lipides.

L'action sur la ponte porte essentiellement sur la précocité, l'âge au premier œuf étant retardé d'environ io jours.

Chez les coqs, il y a diminution de la quantité de spermatozoïdes récoltés et le sperme a une motilité plus faible que chez les témoins. Cette différence au niveau du sperme n'a pas été retrouvée au niveau du testicule. Le taux d'androgènes approché par la taille de la crête semble aussi plus faible chez les sourds.

La fréquence des accouplements des poules sourdes est augmentée.

\section{INTRODUCTION}

Parmi les moyens utilisables pour améliorer la productivité des animaux domestiques, on peut penser à modifier l'environnement sensoriel. Par exemple, on a déjà utilisé la lumière colorée pour limiter le picage et améliorer la croissance du poulet. L'environnement acoustique peut être une autre voie d'approche.

Les travaux de DAPORTA (1967) ont montré que la surdité provoquait, chez la poule élevée en batterie, une augmentation du nombre d'œufs pondus par poule à l'entrée en ponte. Bien que non spectaculaires, ces effets pouvaient être intéressants au point de vue pratique. Favino, Scoz et TréCaTE (I97I) ont montré que les stimulations acoustiques chez 1'Homme provoquaient une sécrétion accrue d'hormones somatotropes. D'autre part, nous avons mis en évidence (FAURE, 1973) l'influence 
de la surdité sur le comportement social. Or, les phénomènes sociaux retentissent chez la Poule aussi bien sur la ponte (SIÉGEL, I959) que sur le comportement sexuel (GUHL. et WARren I946, GuHL I949), ou sur la croissance pondérale (Gover et al I972).

La surdité peut donc agir sur les performances zootechniques, soit directement, soit par l'intermédiaire du comportement social, aussi bien sur la croissance que sur le développement sexuel. C'est ce que nous avons étudié au cours de l'expérience présentée ici.

\section{MATÉRIEI E'T MÉTHODES}

\section{A. - Animaux et méthodes d'élevage}

Les animaux sont issus d'un croisement triple de type ponte (M/5I9) d'origine raciale complexe (Marans, Wyandotte blanche, Rhode-Island rouge). Ce sont ceux pour lesquels nous avons étudié le comportement agonistique (FAURE, I973). Ils proviennent de deux lots éclos les

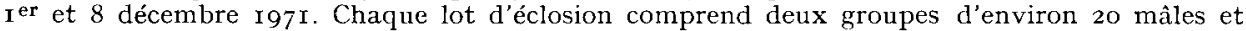
Ioo femelles, les animaux de l'un des groupes étant rendus sourds à l'âge de 8 jours par la méthode d'extirpation de la cochlée décrite par Konishi (1963) (pour plus de détails, voir FAURE, I973) Les animaux ont été élevés en poussinière jusqu'à la ${ }_{1} 5^{\mathrm{e}}$ semaine, puis en poulailler jusqu'à la $5^{2}$ semaine, avec un tiers de la surface occupée par la fosse à déjection. Les animaux ont été soumis au cycle lumineux naturel jusqu'en juin puis maintenus au rythme de 16 heures de lumière par jour (5 h-2I h)

\section{B. -- Mesures effectuées}

\section{Croissance.}

Pour tous les animaux, le poids a été mesuré à $4,8,20,28,36$ et 52 semaines; de plus, à 36 semaines il a été fait une mesure de la longueur du tarse (indicateur de la croissance du squelette). A 52 semaines, 20 animaux de chaque sexe et de chaque traitement ont été sacrifiés afin de peser le tissus adipeux abdominal (indicateur de l'état d'engraissement selon DELPECH et RICARD, I965).

\section{Développement sexuel.}

La ponte a été contrôlée en nids trappe dès l'entrée en ponte. Les œufs ont été pesés pendant les $34^{\mathrm{e}}$ et $35^{\mathrm{e}}$ semaines d'âge, et mis en incubateur pour mesurer le taux d'éclosion pendant les semaines 34 à 37 .

Chez les coqs, la taille de la crête a été utilisée comme indice du taux d'androgènes. Les crêtes sont de deux types, simples et rosacées. La mesure utilisée pour les crêtes simples est la longueur multipliée par la hauteur et pour les crêtes rosacées la longueur multipliéc par la largeur. Les valeurs ont été ensuite transformées en écarts réduits pour pouvoir traiter ensemble les deux types.

La quantité et la qualité du sperme récolté ont été étudiées par la méthode décrite par Petirjean (I965) et Petitjean et de Reviers (1972), c'est-à-dire mesure du volume, de la concentration du sperme par la densité optique et cle la motilité des spermatozoïdes par un appareillage électronique. Une pesée des testicules a été faite sur les coqs abattus à 52 semaines et le nombre de spermatides rondes a été compté dans des coupes transversales de tube séminifère (DE REvrers I97 I $a$ et $b$ ).

\section{Mesures de comportement.}

Les mesures de comportement sexuel ont été faites par observation des animaux à travers une glace sans tain pendant les deux premières heures d'activité, le matin de 5 à $7 \mathrm{~h}$. Seules les copulations apparemment réussies étaient notées. Trois séries d'observations ont été faites : en mai, juillet et novembre. D'autre part, il a été fait en août une série d'observations sur des coqs sourds avec poules témoins et coqs témoins avec poules sourdes.

Pour expliquer les résultats des observations de comportement sexuel, nous avons mesuré le rythme de descente au sol des poules (les poules passent la nuit sur les perchoirs et sur le caillebotis recouvrant la fosse à déjections). Pour cela, nous avons fait une série de photographies (une toutes les 5 minutes, I6 h par jour) d'une partie du sol du poulailler et compté sur les photographies le nombre de poules au sol. 


\section{RÉSULTATS}

\section{I. - Croissance et engraissement}

L'évolution du poids vif des différents groupes d'animanx est indiquée dans la figure I. Les résultats obtenus sur l'échantillon de 20 animaux de chaque sexe et de chaque traitement abattus à un an sont donnés dans le tableau $\mathbf{I}$.

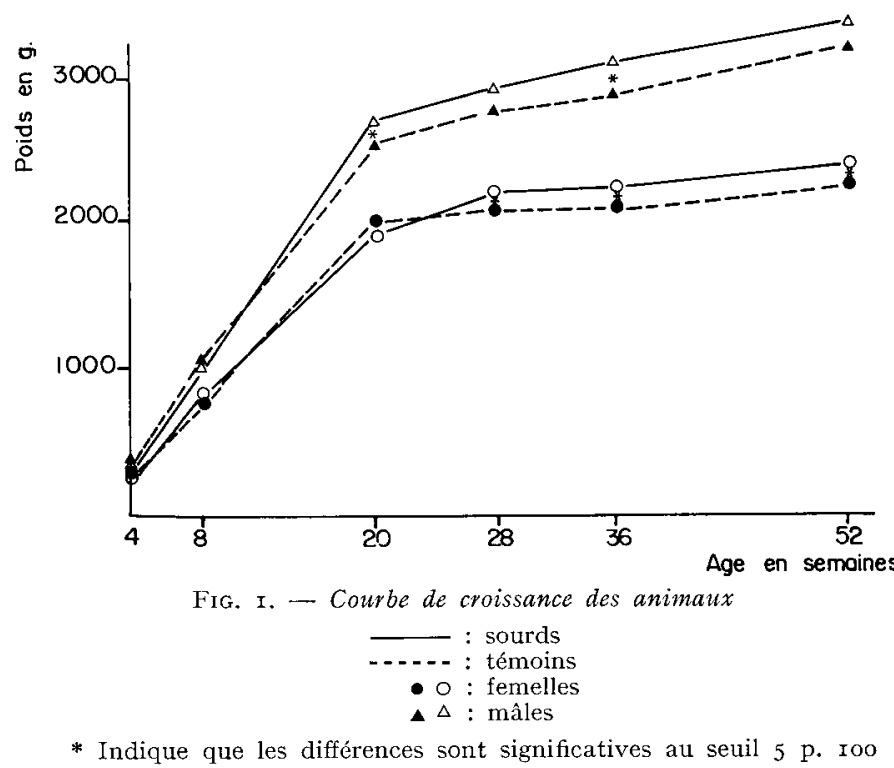

La courbe de croissance (fig. I) montre qu'à partir de 20 semaines pour les coqs et 28 semaines pour les poules, les sourds sont plus lourds que les témoins et que la différence est significative (sauf à 28 et $5^{2}$ semaines pour les coqs).

La mesure de longueur du tarse faite à 36 semaines n'a montré aucune différence entre sourds et témoins ( $\hat{o}$ sourds $129,5 \mathrm{~mm}$; $\partial^{\lambda}$ témoins $\mathrm{I} 29,6 \mathrm{~mm}$; 우 sourdes I04,9 $\mathrm{mm}$; + témoins $104,4 \mathrm{~mm}$ ).

A 52 semaines d'âge, le poids du tissus adipeux abdominal est plus élevé chez les animaux sourds. Mais la différence n'est significative que chez les coqs (tabl. I).

\section{2. - Développement sexuel}

Les résultats concernant la ponte sont consignés dans le tableau 2 . On constate que la longueur des séries, la distance entre séries, le nombre d'œufs pondus à I an par poule à l'entrée en ponte, la mortalité de o à I an ne sont pas affectés par la surdité. Par contre, la précocité, mesurée par l'âge au I ${ }^{\text {er }}$ œuf, est retardée chez les poules sourdes. Le poids des oufs est augmenté et le pourcentage d'œufs doubles ou cassés est plus faible. 


\section{TABLEAU I}

Caractéristiques de croissance et d'engraissement $(n=20$ par sexe et par traitement)

\begin{tabular}{|c|c|c|c|c|c|c|}
\hline & & \multicolumn{2}{|c|}{ Sourds } & \multicolumn{2}{|c|}{ Témoins } & \multirow{2}{*}{$\begin{array}{l}\text { Signification } \\
\text { des différences } \\
(t \text { de Student })\end{array}$} \\
\hline & & $\bar{x}$ & $s$ & $\bar{x}$ & $s$ & \\
\hline \multirow{2}{*}{$\hat{\sigma}$} & Poids à 52 semaines en $g$ & 3256 & 382 & 3119 & 336 & NS $\left({ }^{1}\right)$ \\
\hline & $\begin{array}{l}\text { Poids du tissu adipeux } \\
\text { abdominal en } \mathrm{g}\end{array}$ & 49,7 & 38,5 & 20,3 & 19,0 & $* *$ \\
\hline \multirow{2}{*}{ 우 } & Poids à 52 semaines en $g$ & 2304 & 207 & 2221 & 247 & NS \\
\hline & $\begin{array}{l}\text { Poids du tissu adipeux } \\
\text { abdominal en } g\end{array}$ & 111,2 & 40,6 & 100,3 & 31,9 & NS \\
\hline
\end{tabular}

(1) NS : différence non significative au seuil 5 p. 100.

* : différence significative au seuil 5 p. 100.

**: différence significative au seuil 1 p. 100.

***: différence significative au seuil 1 p. 1000.

TABLEAU 2

Caractéristiques de productivité des poules

\begin{tabular}{|c|c|c|c|c|c|}
\hline & \multicolumn{2}{|c|}{ Sourds $(n=180)$} & \multicolumn{2}{|c|}{ Témoins ( $n=180$ ) } & \multirow{2}{*}{$\begin{array}{l}\text { Signification des } \\
\text { différences }\left({ }^{4}\right)\end{array}$} \\
\hline & $\bar{x}$ ou \% & $s$ & $\vec{x}$ ou $\%$ & $s$ & \\
\hline Age au $1^{\text {er }}$ couf en jours $\ldots . .$. & 162 & 10 & 152 & 8 & $* * * a$ \\
\hline Poids des ceufs en $g \quad \ldots \ldots \ldots \ldots$ & $5 / 4,8$ & 3,8 & $53,{ }^{\prime}$ & 4,2 & $* * * a$ \\
\hline P. 100 d'œufs cassés $(1)$ & 3,9 & - & 5 & - & $* * * b$ \\
\hline P. 100 d'œufs doubles (1)... & 3,0 & — & $r_{4}, 9$ & - & $* * * b$ \\
\hline Longueur des séries $\left({ }^{2}\right) \ldots$ & 77,6 & 32,0 & 72,0 & 31,3 & $\mathrm{NS}^{a}$ \\
\hline Longueur des latences $\left({ }^{3}\right)$ & 15,5 & $1 /, 7$ & 15,2 & 9,9 & $\mathrm{NS}^{\alpha}$ \\
\hline Nombre d'oeufs à 1 an d'âge . . . . & 164,1 & 40,5 & $16^{\prime}, 0$ & 37,4 & NSa \\
\hline Mortalité à 1 an d'âge en p. $100 \ldots$ & 6,8 & - & 5,3 & 一 & $\mathrm{NS}^{b}$ \\
\hline
\end{tabular}

(1) P. 100 d'œufs cassés ou doubles calculés sur les 10 premières semaines de ponte de chaque troupeau.

$\left({ }^{2}\right)$ Longueur des séries : nombre moyen d'œufs pondus par poule pendant les 10 premières séries.

(3) Longueur des latences : durée moyenne en jours de la somme des 10 premières latences entre séries.

(4) $a$ : différences testées par le test $t$ de Student;

$b$ : différences testées par le test du $\chi^{2}$. 
Chez les coqs, l'évolution avec l'âge de la surface de la crête est représentée par la figure 2. On voit que la surface de la crête est toujours infétieure quel que soit 1'âge, chez les coqs sourds (significatif seulement à 20 semaines). Les caractéristiques du sperme sont indiquées dans le tableau 3. Le poids des testicules, le volume de sperme récolté et l'activité testiculaire (nombre de spermatides rondes par coupe transversale de tube séminifère) ne sont pas affectés par la surdité ; par contre la densité optique (et le nombre de spermatozoïdes récoltés) est plus élevée chez les normaux ainsi que la motilité du sperme.

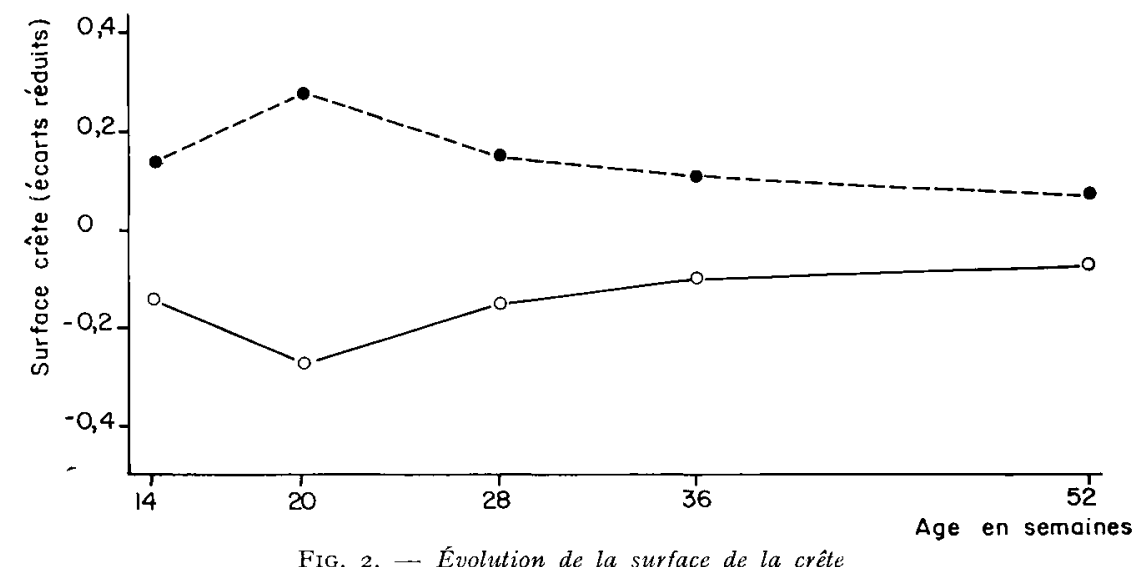

(Crête simple, hauteur $\times$ longueur, crête rosacée : largeur $\times$ longueur) trait plein : sourds, tirets : témoins. Les valeurs mesurées ont été transformées en écarts réduits de manière à pouvoir cumuler les résultats observés pour les deux types de crêtes

Les taux d'éclosion, également indiqués dans le tableau 3, ne sont pas affectés. Il faut noter d'une part que, pour les deux traitements, le taux d'éclosion et la qualité du sperme sont bons et que d'autre part, la qualité du sperme n'est qu'une des composantes de la fertilité et qu'en insémination naturelle le comportement joue aussi un grand rôle.

\section{3. - Comportement sexuel et rythme d'activité}

Les résultats des observations sont rapportés dans le tableat1 4 . On peut constater que les sourds sont toujours significativement supérieurs aux témoins pour le nombre de copulations observées. De plus, ces différences s'accentuent quand les animaux vieillissent.

Pour déterminer le rôle joué par chaque sexe dans ces différences entre traitements, nous avons dans l'un des lots changé les coqs (les coqs témoins mis avec les poules sourdes et inversement). Cette expérience a été réalisée peu après la deuxième série d'observations du tableau 4. Dans le cas des coqs sourds avec poules témoins, le nombre moyen de copulations en 2 heures est de I,5 alors qu'il est de I9,3 dans le cas des coqs témoins placés avec des poules sourdes. Les différences observées correspondent donc à des différences de comportement des femelles. D'autre part, 1'observation des animaux nous a permis de constater que les femelles sourdes descendent 
TABLEAU 3

Mesures de sperme et taux d'éclosion

\begin{tabular}{|c|c|c|c|c|c|}
\hline & \multicolumn{2}{|c|}{ Sourds $(n=40)$} & \multicolumn{2}{|c|}{ Témoins $(n=40)$} & \multirow{2}{*}{$\begin{array}{l}\text { Signification des } \\
\text { différences }\end{array}$} \\
\hline & $\bar{x}$ ou $\%$ & $s$ & $\bar{x}$ ou $\%$ & $s$ & \\
\hline Densité optique $\ldots \ldots \ldots$ & 28 & 7 & 39 & 7 & $* * * a$ \\
\hline Volume en $\mathrm{ml} \ldots$. & 0,72 & 0,12 & 0,77 & 0,15 & $\mathrm{NS}^{a}$ \\
\hline $\mathrm{N} \mathrm{Spz}\left({ }^{1}\right) \ldots \ldots$ & 2054 & 693 & 3001 & 1081 & $* * a$ \\
\hline Motilité (écarts réduits)...... & $-1,49$ & 3,79 & 1,68 & 2,80 & $* * a$ \\
\hline Poids testiculaire en $g$. & 27,7 & 7,2 & 27,6 & 8,2 & $\mathrm{NS}^{a}$ \\
\hline SpdR/CTTS $\left({ }^{2}\right) \quad \ldots \ldots \ldots$ & 276 & 49 & 272 & 50 & $\mathrm{NS}^{a}$ \\
\hline Taux d'éclosion en p. $100 \ldots$ & 90,3 & & 90,6 & & $\mathrm{NS}^{b}$ \\
\hline
\end{tabular}

(1) NSpz : Densité optique $\times$ volume. Mesure approchée du nombre de spermatozoïdes récoltés.

$\left({ }^{2}\right)$ SpdR/CTTS : Nombre de spermatides rondes par coupe transversale de tube séminifère.

\section{TABLEAU 4}

Observation du comportement sexuel

(nombre moyen de copulations pendant les deux premières heures d'activité le matin)

\begin{tabular}{c|c|c|c}
\hline \hline $\begin{array}{c}\text { Epoque des } \\
\text { observations }\end{array}$ & Sourds & Témoins & $\begin{array}{c}\text { Signification des } \\
\text { différences }\end{array}$ \\
\hline Mai & 12,8 & 8,5 & $*$ (1) \\
\hline Juillet & 17,4 & 4,3 & $* *$ \\
\hline Novembre & 22,2 & 2,7 & $* *$ \\
\hline
\end{tabular}

(1) Différences testées par le test U de Mann et Whitney (Sregel, 1956)

plus tôt des perchoirs ou du caillebotis recouvrant la fosse à déjections que les femelles témoins. Ces observations ont pu être quantifiées par le dépouillement de photographies et les résultats en sont illustrés par la figure 3 .

On constate que le matin aux heures où sont faites les observations ( 5 à $7 \mathrm{~h}$ ), le nombre de poules sourdes est significativement supérieur au nombre de poules normales au sol et que les mêmes différences se retrouvent de I3 à I8 heures. Ces deux périodes de la journée correspondent au maximum d'activité sexuelle (PETITJEAN I965 et observations personnelles). L,es poules sourdes sont donc plus actives sexuellement et se trouvent au sol au moment du maximum d'activité sexuelle des coqs. 


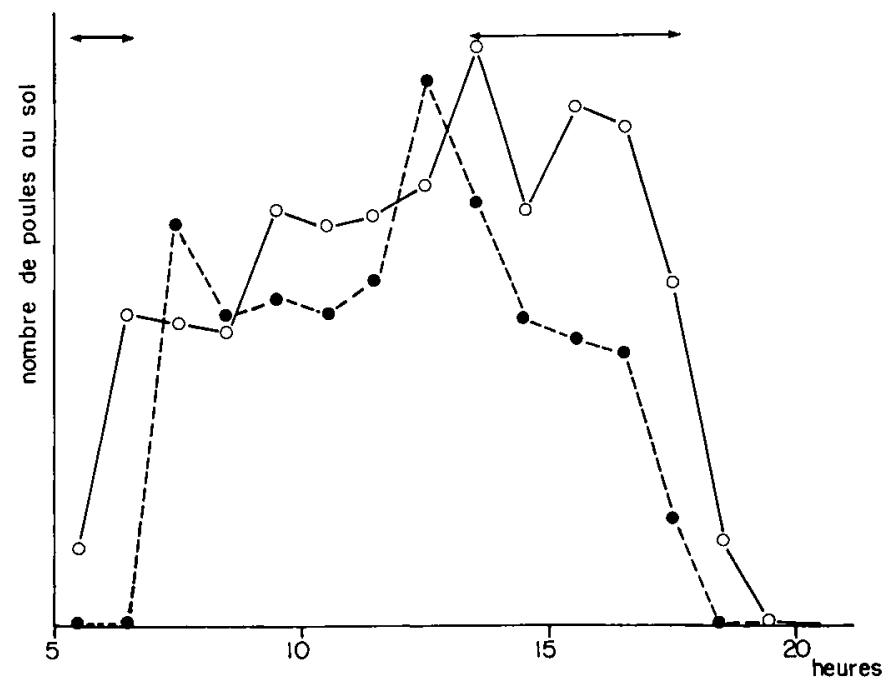

FIG. 3. - Nombre de poules au sol en fonction de l'heure

(Traits pleins : sourdes, tirets : témoins). Le signe $\longleftrightarrow$ indique pour les points recouverts que les différences sont significatives au seuil $5 \mathrm{p}$. Ioo ( $t$ de Student)

\section{DISCUSSION}

L'effet de la surdité se traduit dans les deux sexes par une augmentation du poids à partir de 20 semaines chez les sourds. Cette augmentation du poids n'a pu être reliée à une augmentation de la taille, estimée par la longueur de la patte. Il n'y a donc aucune influence, mesurable par cette méthode, de la surdité sur la sécrétion d'hormone somatotrope. Le poids plus élevé des animaux sourds pourrait être lié à un engraissement supérieur. Mais notre échantillon ne permet pas de conclure avec certitude : le nombre d'animaux abattus était trop faible pour mettre en évidence une différence des poids chez les poules (alors que cette différence est significative si on considère l'ensemble du troupeau). Cependant si le poids de tissus adipeux abdominal n'est significativement supérieur que chez les coqs sourds, on retrouve la même tendance chez les poules et l'absence de signification peut être due à la faiblesse de l'échantillon.

L'entrée en ponte des poules sourdes est retardée de Io jours en moyenne. On peut penser que le pourcentage plus faible d'œufs doubles ou cassés et le poids des œufs plus élevé sont une conséquence de ce retard à 1'entrée en ponte. Malgré le retard de précocité dù à la surdité, à un an d'âge le nombre d'œufs pondus est le même pour les deux traitements.

Nous n'avons donc pas retrouvé les résultats de DAPOR'TA (I967) concernant l'augmentation de la ponte et la diminution de la mortalité. Il faut cependant remarquer que la technique opératoire est différente. Alors que nous avons utilisé l'ablation de la cochlée, DAPor'Ta utilisait la perforation du tympan, c'est-à-dire une méthode permettant une récupération de l'audition. D'autre part, les conditions d'élevage étaient différentes (élevage en batteries dans le cas des travaux de DAPOR'TA, élevage 
au sol dans le cas présent). Dans la mesure où le développement de la crête est un bon indice du taux d'androgènes, on peut dire que les coqs sourds ont un développement sexuel plus faible mais aussi plus lent puisque c'est à 20 semaines que les différences sont les plus grandes. Cependant à un an d'âge le poids du testicule, l'activité testiculaire mesurée par la méthode de DE REVIERS et le volume de sperme récolté ne sont pas affectés par le traitement. Par contre, l'examen du sperme fait apparaître une concentration en spermatozoïdes et une motilité des spermatozoïdes plus faible. En pratique, les différences observées n'ont pas de conséquence sur le taux d'éclosion mais ceci peut être dû à la bonne qualité du sperme dans les deux cas et aussi à la différence de comportement sexuel mise en évidence chez les poules.

\section{CONCLUSION}

Il semble difficile d'expliquer l'ensemble des effets observés par le seul effet de la suppression d'un mode de communication. Il est plus probable que 1'action primaire se fait au niveau du système nerveux central (formation réticulée et phénomènes de vigilance?) et par là peut agir sur le système endocrinien des animaux ; ce qui expliquerait aussi bien l'action sur le comportement que sur la croissance ou sur le développement sexuel.

Sur le plan zootechnique la surdité, si elle ne présente pas d'inconvénients majeurs, ne présente pas non plus d'avantages très marqués. D'autre part, la durée de 1'opération en limiterait l'application pratique. I1 pourrait cependant être intéressant de rechercher un gène de surdité afin de voir si par cette voie on obtiendrait les mêmes effets et aussi s'il n'y aurait pas alors une possibilité d'application.

Reçu pour publication en novembre 1973.

\section{REMERCIEMENTS}

Je tiens à remercier MM. Ricard, Petitjean et de Reviers et leurs équipes qui ont bien voulu se charger respectivement des mesures de croissances, des mesures de spermiologie, et des mesures d'activité testiculaire.

\section{SUMMARY}

\section{EFFECT OF DEAFNESS ON BODY DEVELOPMEN'T \\ AND REPRODUCTIVE PERFORMANCE IN GALLUS DOMESTICUS}

The influence of deafness on various zootechnical characteristics has been studied in a strain of medium size layers.

Deafness has an effect on growth of cocks and hens by increasing adult weight but the difference cannot be imputed only to an accumulation of lipids.

The effect on laying pattern mainly concerns the precocity, the age at first egg being delayed by about ten days. 
When compared with controls, a reduction of the quantity of spermatozoa and a lower quality of semen are found in deaf cocks. But this difference concerning sperm characteristics has not been found at the testes level. Rate of androgens seems to be poorer for deaf birds. Number of complete mating of deaf hens is increased.

\section{RÉFÉRENCES BIBLIOGRAPHIQUES}

Daporta M.-R., I967. The suppression of the sense of hearing in laying hens, J. Agric. Univ. P. Rico, 51, 267-268.

Delpech P., Ricard F.-H., I965. Relations entre les dépôts adipeux viscéraux et les lipides corporels chez le poulet. Ann. Zootech., 14, I8I-I89.

FAURE J.-M. Influence de la surdité sur le comportement social de Gallus Domesticus. Rev. Comp. Animal, 7, 253-258.

Favino A., Scoz R., Trécate G., r97i. Effetti della stimulazione acustica sulla secrezione di ormone somatropo umano.' Boll. Soc. Ital. Biol. Sper., 47, 704-708.

Gover R.-M., Anderson D.-L., Damon R.-A., Ruggles L.-H., 1972. The effects of bird density, dietary energy, light intensity and cage level on the reproductive performance of heavy type of chickens in wire cages. Poult. Sci., 51, 565-575.

GuHL A.-M., 1949. Heterosexual dominance and mating behaviour in chickens. Behaviour, 2, 106-120-

Guhl A.-M., Warren D. C., I946. Number of offsprings sired by cockerels related to social domnance in chickens. Poult. Sci., 25, 460-472.

Konishr M., I963. The role of auditory feedback in the vocal behaviour of the domestic fowl. Z. Tier. psychol., 20, 349-367.

Petitjean M.-J., I965. Recherches sur l'estimation du pouvoir fécondant des coqs. Thèse des arts et métiers (non publié).

Petitjean M.-J., Reviers M. de, I972. Effets du gène de nanisme dw sur la maturité sexuelle des coqs. 4th Europ. Poult. Conf. London, 569-575.

Reviers M. de, x97r a. Le développement testiculaire chez le Coq. I. Croissance pondérale des testicules et développement des tubes séminifères. Ann. Biol. Anim. Bioch. Biophys., 11, 519-530.

Reviers M. de, I97i $b$. Le développement testiculaire chez le Coq. II. Morphologie de l'épithélium séminifère et établissement de la spermatogenèse. Ann. Biol. Anim. Bioch. Biophys., 11, 53r-546.

SiEGEL H.,-S., I959. Egg production characteristics and adrenal function in white leghorns confined at different floor space levels. Poult. Sci., 38, $893^{-898}$.

SiEgeL S., 1956. Non parametric statistics for the behavioral science. Mac Graw-Hill, New York. 